\title{
THE INFLUENCE OF AXIAL MICROGRAVITY ON THE BREAKAGE OF AXISYMMETRIC SLENDER LIQUID BRIDGES
}

\author{
J. MESEGUER \\ Laboratorio de Aerodinámica. ETSI Aeronáuticos. Universidad Politécnica, Madrid, Spain
}

\begin{abstract}
The dynamics of inviscid. axisymmetric liquid bridges permits a simplified treatment if the bridge is long enough. Under such condition the evolution of the liquid zone is satisfactorily explained through a non-linear one-dimensional model. In the case of breaking. the one-dimensional model fails when the neck radius of the liquid column is close to zero; however. the model allows the calculation of the time variation of the liquid-bridge interface as well as of the fluid velocity field and. because the last part of the evolution is not needed, the overall results such as the breaking time and the volume of each of the two drops resulting after breakage can be calculated. In this paper numerical results concerning the behavior of cylindrical liquid bridges subjected to a small axial gravitational field are presented.
\end{abstract}

\section{Introduction}

The near-zero gravity environment of an orbiting space station offers unique opportunities for improving the knowledge on the processes involved in crystal growth by the floating zone technique. In such an environment the constraints imposed by gravity on the dimensions of a liquid bridge are partially removed. However, the maximum length of a liquid bridge, even in zero gravity condition, is limited by stability phenomena: it is well known from the studies of Plateau and Rayleigh that the maximum stable length of a cylindrical, circular cross section, liquid bridge at rest is reached when the ratio of the zone length to the undisturbed diameter exceeds the value $\pi$. This maximum stable length is reduced when a gravitational field acts along the liquid-bridge axis and increasing attention is being paid in the scientific literature to analysing the stability limits of liquid bridges under such circumstance [1-6].

The study of real molten liquid bridges involves a large variety of phenomena which increase the difficulty of the problem: melting and freezing fronts, melt properties strongly temperature-dependent, imposed disturbances (either accidentally or intentionally), etc. Thence, several plausible simplifications must be introduced to get treatable models. When the aim is the study of mechanical aspects of the liquid-bridge problem, leaving apart thermal problems, the simplest approach consists in disregarding phase changes, considering a liquid bridge, with uniform and constant properties, filling the gap between two solid discs.

The problem to be solved, as fig. 1 shows, concerns the evolution of an inviscid, slender liquid bridge having cylindrical volume, held by surface tension forces between two parallel, coaxial, equal diameter solid discs, with anchored edges, when disturbed by the action of a small gravitational field parallel to the axis of the bridge. To perform this study the following assumptions are introduced:

(1) Internal movement in the liquid bridge is only due to the capillary pressure gradients generated by the deformation of the interface and the hydrostatic pressure gradient.

(2) The gas atmosphere surrounding the liquid does not affect the dynamics of the liquid bridge. (3) Inertia forces, due to a non-uniform displacement of the liquid bridge as a whole, are absent.

(4) Since gravity acts parallel to the bridge axis, 
only axisymmetric configurations are considered. Thence, the problem is independent of the azimuthal coordinate.

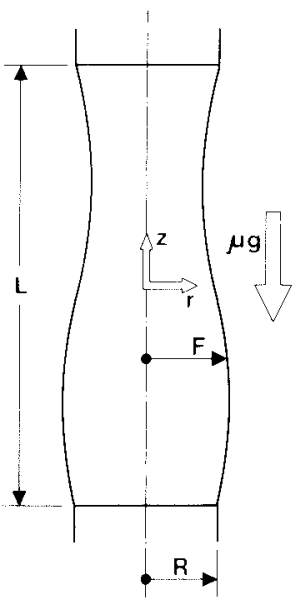

Fig. 1. Geometry and coordinate system for the liquid bridge.

(5) The liquid density, $\rho$, as well as the liquid-gas surface tension, $\sigma$, are uniform and constant.

\section{The one-dimensional slice model}

The slice model is based on a one-dimensional theory similar to the one used by Lee [7] in the study of the dynamics of capillary jets.

The main simplification introduced by the slice model is that the axial velocity depends only upon the axial coordinate and the time. Under that assumption the set of nondimensional differential equations and boundary conditions for the axisymmetric, non-rotating inviscid flow in cylindrical coordinates are drastically reduced [8]. Since the axial velocity does not depend on the radial coordinate, the radial momentum equation becomes decoupled from the other equations and the study of the evolution of the liquid bridge may be accomplished by solving only the continuity equation and the axial momentum equation:

$\frac{1}{r} \frac{\partial}{\partial r}(r U)+\frac{\partial W}{\partial z}=0$

$\frac{\partial W}{\partial t}+W \frac{\partial W}{\partial z}=-\frac{\partial P}{\partial z}$, where $P$, which takes into account the capillary and hydrostatic pressures, and does not depend on $r$, is related to the shape of the interface, whose equation is $r=F(z, t)$, through:

$$
\begin{aligned}
P= & \frac{1}{\left[1+(\partial F / \partial z)^{2}\right]^{3 / 2}} \\
& \times\left[\frac{1+(\partial F / \partial z)^{2}}{F}-\frac{\partial^{2} F}{\partial z^{2}}\right]+\text { Bo } z .
\end{aligned}
$$

To write down these expressions all lengths have been made dimensionless with the radius of the discs, $R$, and the radial and axial velocities, $U$ and $W$ respectively, with $\sqrt{\sigma /(\rho R)}$. Time has been made dimensionless with $\sqrt{\rho R^{3} / \sigma}$ and the reduced gauge pressure with $\sigma /(\rho R)$. Finally, $\mathrm{Bo}$ is the static Bond number, $\mathrm{Bo}=\rho g R^{2} / \sigma$, where $g$ is the acceleration due to axial microgravity.

The boundary conditions to be met within the one-dimensional inviscid slice model are for the axial velocity to be zero at the discs:

$W( \pm \Lambda, t)=0$.

$\Lambda=L /(2 R)$ being the slenderness of the liquid bridge, and for the interface to remain anchored to the disc edges:

$F( \pm \Lambda, t)=1$.

One more boundary condition must be added, expressing that the interface is a material surface. since the mass flow rate trough it is zero:

$\frac{\partial F}{\partial t}-U+W \frac{\partial F}{\partial z}=0$.

Finally, the formulation will be completed by fixing suitable initial conditions:

$F(z, 0)=F_{0}(z), \quad W(z, 0)=W_{0}(z)$,

where $F_{0}(z)$ must satisfy the condition of cylindrical volume:

$\int_{-.1}^{+\lambda} F_{0}^{2} \mathrm{~d} z=2 \Lambda$

To eliminate the velocity $U$ from the previous formulation, we may substitute for (1) and (6) a new equation expressing the overall mass con- 
servation in each cross-section. By application of the continuity principle to a control volume bounded by two contiguous cross-sections and the corresponding interface, the following result is obtained:

$\frac{\partial F^{2}}{\partial t}+\frac{\partial}{\partial z}\left(F^{2} W\right)=0$.

The problem is more conveniently adapted for calculations by choosing as computing variables $S=F^{2}$ and $Q=F^{2} W$, which are, respectively, proportional to the cross sectional area and to the axial momentum of a slice. By using these new variables the continuity equation (9) and axial momentum equation ( 2 ) become:

$\frac{\partial S}{\partial t}+\frac{\partial Q}{\partial z}=0$

$\frac{\partial Q}{\partial t}+\frac{\partial}{\partial z}\left(\frac{Q^{2}}{S}\right)=-S \frac{\partial P}{\partial z}$.

where $P$ is now related to $S$ through:

$$
\begin{aligned}
P= & \frac{2}{\left[4 S+(\partial S / \partial z)^{2}\right]^{1 / 2}} \\
& \times\left[1-\frac{2 S \partial^{2} S / \partial z^{2}-(\partial S / \partial z)^{2}}{4 S+(\partial S / \partial z)^{2}}\right]+\text { Bo } z .
\end{aligned}
$$

Boundary conditions are:

$S( \pm \Lambda, t)=1, \quad Q( \pm \Lambda, t)=0$,

and initial conditions:

$S(z, 0)=S_{0}(z), \quad Q(z, 0)=Q_{0}(z)$.

The numerical integration of this set of hyperbolic differential equations may be accomplished by using finite difference methods. In this case the problem has been solved by means of the Lax-Wendroff method $[9,10]$ which consists in developing in Taylor series with respect to time the variables $S$ and $Q$, retaining up to $(\Delta t)^{2}$ terms:

$$
\begin{aligned}
S(z, t+\Delta t)= & S(z, t)+\Delta t \frac{\partial}{\partial t} S(z, t) \\
& +\frac{1}{2}(\Delta t)^{2} \frac{\partial^{2}}{\partial t^{2}} S(z, t)+\ldots
\end{aligned}
$$

$$
\begin{aligned}
Q(z, t+\Delta t)= & Q(z, t)+\Delta t \frac{\partial}{\partial t} Q(z, t) \\
& +\frac{1}{2}(\Delta t)^{2} \frac{\partial^{2}}{\partial t^{2}} Q(z, t)+\ldots
\end{aligned}
$$

the time derivatives appearing in (15) being calculated through (10) to (12) in terms of the spatial derivatives. Additional details on computations, mainly those in connection with the boundary conditions (13), can be found in ref. [8] where the evolution of liquid bridges in the case $\mathrm{Bo}=0$ is treated.

\section{Initial conditions}

Computations have been carried out for different values of the slenderness of the liquid bridge, $A$, and different values of the Bond number, Bo, as table 1 shows. For all the cases included in this table the initial conditions were:

$S(z, 0)=1, \quad Q(z, 0)=0$,

that is, at zero time the liquid shape is cylindrical and no velocity field is imposed.

Concerning these initial conditions, obviously when $\mathrm{B} o \neq 0$ the cylinder is not an equilibrium shape, the magnitude of the interface perturbation represented by (16) can be obtained by comparing the cylindrical shapes with the equilibrium ones, which are calculated through an hydrostatic analysis, that is, by solving eq. (3) under the assumption that $P$ is a constant. If the Bond number is small enough the variables involved in the problem may be rewritten as:

$F=1+\epsilon f, \quad P=1+\epsilon p, \quad$ Bo $=\epsilon b$,

with $\epsilon \ll 1$ and $f, p$ and $b$ of order unity. Substituting expressions (17) in eqs. (3), (5) and (8), leaving apart the $\epsilon^{2}$ terms, yields:

$\frac{\partial^{2} f}{\partial z^{2}}+f-p-b z=0$

with boundary conditions:

$f( \pm \Lambda)=0, \quad \int_{-, \Lambda}^{+\Lambda} f \mathrm{~d} z=0$. 
Table 1

Breaking time, $t_{\mathrm{b}}$, and breaking volume, $V_{\mathrm{p}}$, of initially cylindrical liquid bridges having different slendernesses, $A$, for several values of the Bond number, Bo

\begin{tabular}{|c|c|c|c|c|c|c|c|c|}
\hline \multicolumn{3}{|c|}{$\mathrm{Bo}=0.001$} & \multicolumn{3}{|c|}{ Bo $=0.002$} & \multicolumn{3}{|c|}{ Bo $=0.005$} \\
\hline$A$ & $t_{\mathrm{b}}$ & $V_{\mathrm{p}}$ & $\Lambda$ & $t_{b}$ & $V_{\mathrm{p}}$ & $\Lambda$ & $t_{\mathrm{h}}$ & $V_{\mathrm{p}}$ \\
\hline 3.2 & 33.6 & 0.8415 & 3.2 & 27.1 & 0.8404 & 3.14 & 20.8 & 0.8437 \\
\hline 3.14 & 38.4 & 0.8477 & 3.1 & 32.7 & 0.8508 & 3.1 & 21.8 & 0.8481 \\
\hline 3.1 & 45.7 & 0.8516 & 3.05 & 42.5 & 0.8560 & 3.05 & 23.7 & 0.8533 \\
\hline \multirow[t]{2}{*}{3.06} & \multirow{2}{*}{\multicolumn{2}{|c|}{ Oscillation }} & 3.0 & \multirow{2}{*}{\multicolumn{2}{|c|}{ Oscillation }} & 3.0 & 27.3 & 0.8586 \\
\hline & & & & & & 2.95 & Osci & \\
\hline
\end{tabular}

\begin{tabular}{|c|c|c|c|c|c|c|c|c|}
\hline \multicolumn{3}{|c|}{$\mathrm{Bo}=0.01$} & \multicolumn{3}{|c|}{$\mathrm{Bo}=0.02$} & \multicolumn{3}{|c|}{$\mathrm{Bo}=0.05$} \\
\hline$\Lambda$ & $t_{\mathrm{h}}$ & $V_{\mathrm{p}}$ & $\Lambda$ & $t_{\mathrm{h}}$ & $V_{p}$ & $\Lambda$ & $t_{\mathrm{b}}$ & $V_{\mathrm{p}}$ \\
\hline 3.2 & 15.3 & 0.8325 & 3.2 & 11.6 & 0.8216 & 3.2 & 7.9 & 0.7945 \\
\hline 3.1 & 16.1 & 0.8431 & 3.1 & 12.0 & 0.8331 & 3.0 & 8.1 & 0.8163 \\
\hline 3.0 & 17.7 & 0.8542 & 3.0 & 12.5 & 0.8460 & 2.8 & 8.5 & 0.8460 \\
\hline 2.9 & 22.5 & 0.8654 & 2.9 & 13.4 & 0.8571 & 2.6 & 9.8 & 0.8758 \\
\hline \multirow[t]{3}{*}{2.85} & \multirow{3}{*}{\multicolumn{2}{|c|}{ Oscillation }} & 2.8 & 15.7 & 0.8697 & 2.55 & 10.9 & 0.8827 \\
\hline & & & 2.75 & 20.0 & 0.8752 & 2.5 & 17.2 & 0.8890 \\
\hline & & & 2.7 & Oscil & & 2.45 & Oscil & \\
\hline
\end{tabular}

The solution of (18) with boundary conditions (19) is:

$f=b\left(z-\frac{\Lambda}{\sin \Lambda} \sin z\right)$,

with $p=0$. Thence, the interface shape is given by:

$F=1+\operatorname{Bo}\left(z-\frac{\Lambda}{\sin \Lambda} \sin z\right)$.

This first order analysis gives the deformation of the interface but not the static stability limit which requires higher order analysis. Vega and Perales [6] have calculated this static stability limit when Bo $\ll 1$, obtaining:

$\Lambda_{\mathrm{cr}}=\pi\left[1-\frac{3}{2}\left(\frac{3}{2} \mathrm{Bo}^{2}\right)^{1 / 3}\right]$,

$\Lambda_{\text {cr }}$ being the maximum stable slenderness of a liquid bridge having cylindrical volume for a given Bond number.

The magnitude of the interface deformation at the stability limit can be measured by the parameter $d_{\mathrm{n}}=1-F_{\mathrm{m}}$, where $F_{\mathrm{m}}$ stands for the minimum value of $F$ which is reached at:

$$
z_{\mathrm{m}}=\cos ^{-1}\left(\frac{\sin A}{\Lambda}\right)
$$

thus, the interface deformation at the static stability limit is:

$$
d_{\mathrm{n}}=\sqrt{\frac{2}{3}\left[\frac{2}{3}\left(1-\frac{\Lambda_{\mathrm{cr}}}{\pi}\right)\right]^{3}}\left(z_{\mathrm{m}}-\frac{\Lambda_{\mathrm{cr}}}{\sin \Lambda_{\mathrm{cr}}} \sin z_{\mathrm{m}}\right) .
$$

Fig. 2 shows the variation of $d_{n}$ with the critical slenderness and the Bond number. The meaning of initial conditions (16) is that for each of the liquid bridges considered a perturbation like (20) has been added to the equilibrium shape, being the amplitude of the perturbation given by fig. 2 . Thence, if the Bond number is smaller than that corresponding to the select point $\left(\Lambda, d_{n}\right)$ the liquid bridge will be stable; on the contrary, if $B o$ is larger, breakage will take place.

With respect to the range of values of the Bond number used in the computations, the chosen values of Bo are in accordance with those expected in an earth-orbiting station (like the Spacelab/Shut- 


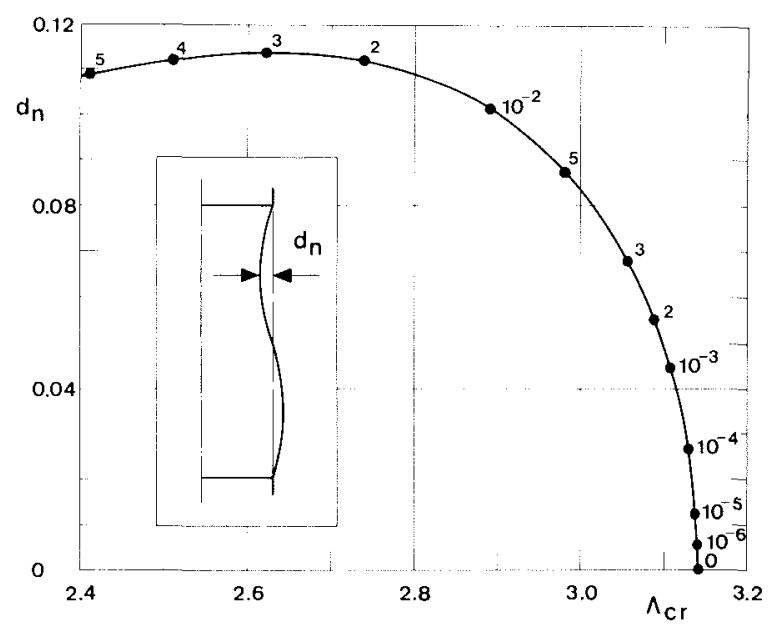

Fig. 2. Deformation of the liquid bridge, $d_{n}$ (defined as sketched in the insert), versus critical slenderness, $A_{\mathrm{ur}_{\mathrm{r}}}$. Numbers on the curve indicate values of the Bond number, Bo.

tle system) or in Plateau simulation in a ground laboratory. In effect, if we consider liquid bridges held between dises with radii of the order of $10^{-2}$ $\mathrm{m}$, and common liquids used in experimentation, such as water or dimethyl silicone, having densities close to $10^{3} \mathrm{~kg} \mathrm{~m}^{-3}$, the value of the surface tension may range, let say, from $10^{-2}$ to $10^{-1} \mathrm{~N}$ $\mathrm{m}^{-1}$; concerning axial acceleration, its maximum value onboard a space platform can be $10^{-3} \mathrm{~m} \mathrm{~s}^{-2}$ (under normal circumstances which do not include crew handling shocks [11]), and thence the Bond number ranges, in this case, from $10^{-3}$ to $10^{-2}$.

On the other hand, in Plateau simulation the Bond number is defined by the difference between the densities of both working-liquid and bath; the maximum value of this difference depends on the desired slenderness of the liquid column, thus, to get liquid bridges having slendernesses close to 3 a limiting value $\Delta \rho / \rho \sim 10^{-3}$ has been quoted $[1,12]$ but this limiting value seems to be inappropriate and the lowest value $\Delta \rho / \rho \sim 10^{-5}$ has been established in a more recent paper [13]. Also in this case typical Bond numbers are close to $10^{-3}$.

\section{Numerical results}

The purpose of computations was to obtain the evolution of the interface shape and the liquid

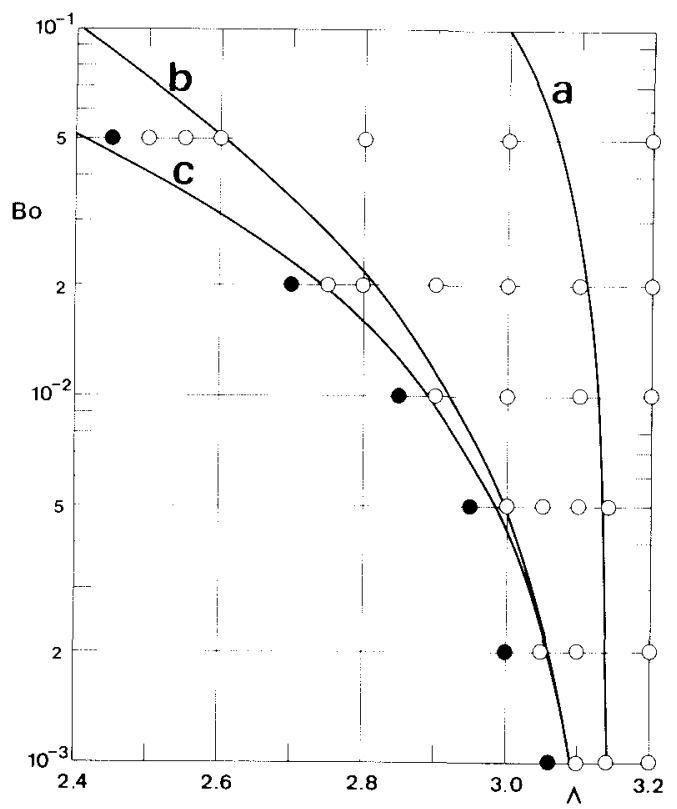

Fig. 3. Static stability limits (Bond number, Bo, versus slenderness. A) of initially cylindrical liquid bridges as given by several investigators: (a) Carruthers and Grasso [1]. (b) Coriell, Hardy and Cordes [2]. (c) Vega and Perales [6]. The circles correspond to the cases numerically solved through the one-dimensional slice model, with indication of the behavior of the liquid bridge: oscillation (closed circles) or breaking (open circles).

velocity field, as well as the breaking time and the volume of the two drops resulting after the breakage (we denote as breaking volume the ratio of the volume of the main drop to the whole liquid bridge volume). Each case numerically solved has been represented by a point in the plane $(\Lambda, \mathrm{Bo})$ : fig. 3 shows this plane, with an indication of the results obtained: breakage (open circles) or oscillation (closed circles). As can be seen there is a defined border between both open and closed circles which splits the plane in two different regions. Points on the right of this border represent unstable configurations, the stable configurations corresponding to the left-hand side. Thus, the border corresponds to the limit of stability.

Obviously, this stability limit cannot be reached numerically, since the evolution time becomes infinite. To compare this result with those obtained from hydrostatic studies the static stability limits as given by several investigators are also shown in 
Table 2

Values of the static stability limit selected from Coriell. Hardy and Cordes [2]

\begin{tabular}{ll}
\hline$A$ & Bo \\
$\pi$ & 0 \\
3.12 & 0.000258 \\
3.10 & 0.000702 \\
3.05 & 0.00239 \\
2.96 & 0.00722 \\
2.92 & 0.0100 \\
2.85 & 0.0162 \\
2.815 & 0.0200 \\
2.60 & 0.0518 \\
2.405 & 0.100 \\
2.155 & 0.200 \\
\hline
\end{tabular}

fig. 3. Curve a was suggested by Carruthers and Grasso [1] and it was found through an analogy with the stability limit of liquid bridges under solid rotation; these authors assume that the stability limit may be expressed as:

$A_{\mathrm{c} r}=\pi(1+\mathrm{Bo})^{-1 / 2}$,

where $\Lambda_{t r}$, as stated above, is the maximum stable

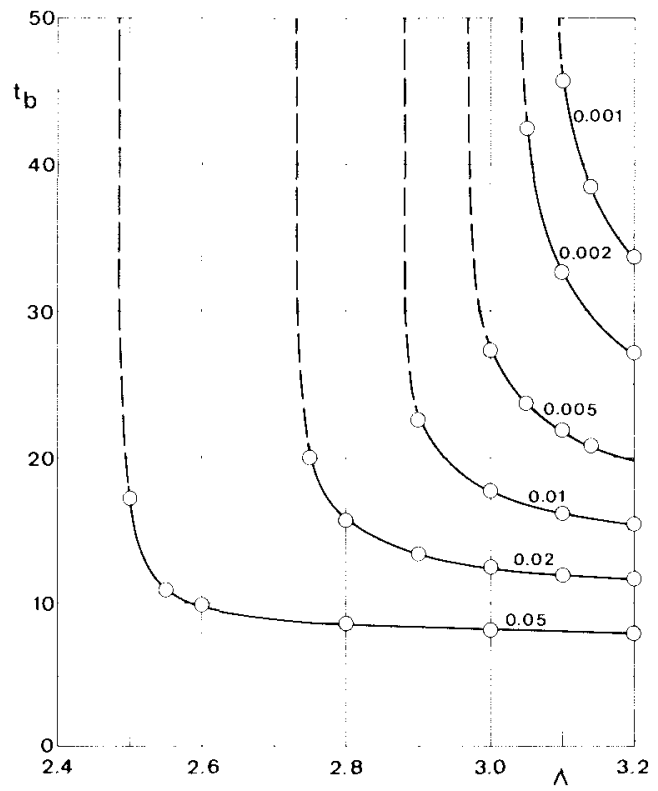

Fig. 4. Breaking time, $t_{h}$, versus slenderness, $A$, of initially cylindrical liquid bridges. Numbers on the curves indicate values of the Bond number. Bo.

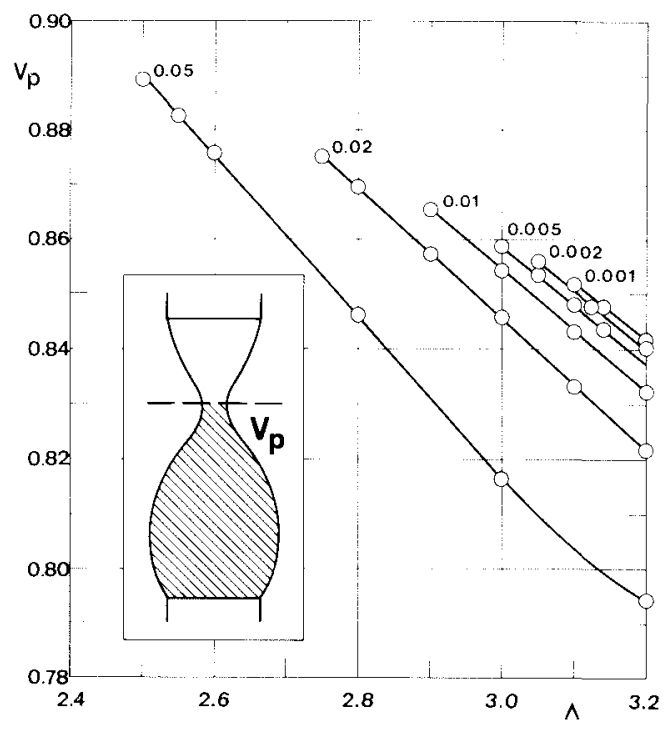

Fig. 5. Breaking volume, $V_{p}$, versus slenderness. 1 , of initially cylindrical liquid bridges. Numbers on the curves indicate values of the Bond number, Bo.

slenderness of a cylindrical volume liquid bridge for the corresponding Bond number.

The stability limits represented by curves $b$ and $c$ have been derived by studying the bifurcation appearing when $\Lambda=\pi$ in the Laplace-Young capillary equation for the shape of axisymmetric liquid bridges. Curve b was numerically obtained by Coriell. Hardy and Cordes [2] which gave the values shown in table 2 . whereas curve c has been analytically calculated by Vega and Perales [6] by using asymptotic expansion methods, obtaining the result already presented in eq. (22).

The results here obtained through the slice model seem to indicate that the criterium settled by Carruthers and Grasso is clearly erroneous. The dynamic analysis gives results which are in accordance with curves $b$ and $c$, although agreement with curve $c$ is better within the range of considered Bond numbers.

With the data compiled in table 1, figs. 4 and 5 have been prepared. In fig. 4 the breaking time. $t_{b}$. versus the slenderness of the liquid bridge is plotted for several values of the Bond number. In each curve $\mathrm{Bo}=$ constant the breaking time increases as the slenderness decreases, and becomes infinite close to a critical value of the slenderness. $A_{c r}$ : for 


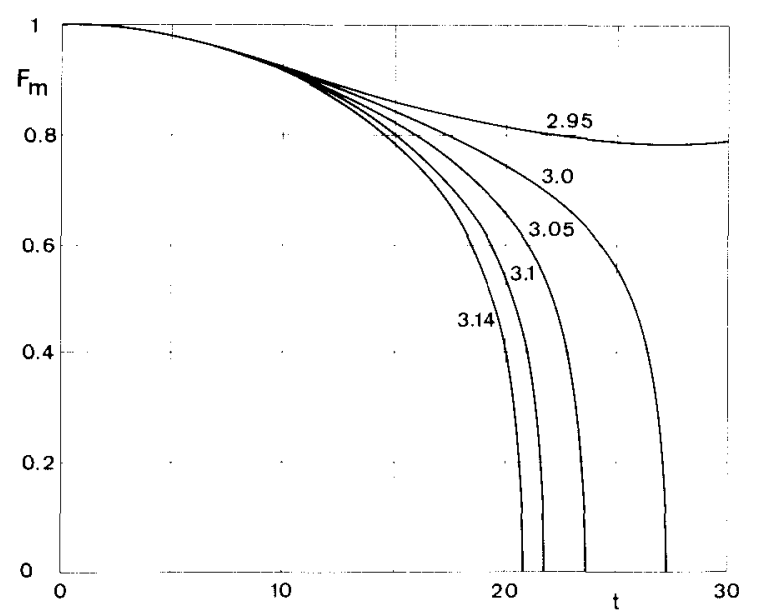

Fig. 6. Variation with time, $t$, of neck radius of the liquid bridge. $F_{\mathrm{m}}$, in the case $\mathrm{Bo}=0.005$. Numbers on the curves indicate values of the slenderness of the liquid bridge, $A$.

smaller values than $\Lambda_{\text {cr }}$ no breakage occurs: the liquid bridge is stable for the imposed initial conditions. The variation of the breaking volume (as defined above) with the slenderness is shown in fig. 5 ; the breaking volume, $V_{\mathrm{p}}$, decreases as both the slenderness of the liquid bridge and the Bond number increase, and seems to vary linearly with A.

The behavior of the liquid bridge during the evolution can be illustrated by plotting the variation with time of the neck radius of the liquid bridge, as fig. 6 shows in the case $\mathrm{Bo}=0.005$ (the behavior for other values of the Bond number is similar). At the beginning the neck radius varies slowly independently of the character of the evolution (oscillation or breakage). After this period, if the slenderness is smaller than the critical one, the neck radius grows and oscillates around some value (that corresponding to the equilibrium shape). On the contrary, if $A$ is greater than $A_{\text {cr }}$ the neck radius continuously decreases, the evolution being more and more fast as the time increases; for instance, if $\Lambda=3$, the liquid bridge spends $94 \%$ of the breaking time to reduce the neck radius from 1 to 0.5 . whereas in the remaining $6 \%$ the neck radius decreases from 0.5 to 0 .

The breaking process can be more easily understood through the balance of the different energies involved. These energies, which have been made dimensionless with $\sigma R^{2}$, are the kinetic energy

$E_{\mathrm{c}}=\frac{\pi}{2} \int_{-\Lambda}^{+\Lambda} F^{2}\left[W^{2}+\frac{1}{8} F^{2}(\partial W / \partial z)^{2}\right] \mathrm{d} z$.

the potential energy

$E_{\mathrm{p}}=\pi \mathrm{Bo} \int_{-A}^{+\Lambda}(z+\Lambda) F^{2} \mathrm{~d} z$,

and the free surface energy

$E_{\mathrm{s}}=2 \pi \int_{-.1}^{+1} F \sqrt{1+(\partial F / \partial z)^{2}} \mathrm{~d} z$

whose values at the starting time, when the shape is a circular cylinder, are: $E_{\mathrm{c} 0}=0, E_{\mathrm{p} 0}=2 \pi \mathrm{Bo} A^{2}$ and $E_{\mathrm{s} 0}=4 \pi \Lambda$.

Let us go through the reasoning with a representative case: fig. 7 shows the energy evolution of a liquid bridge whose slenderness is 3.1 in the case $\mathrm{B}_{\mathrm{O}}=0.002$. At the beginning of the process the hydrostatic pressure gradient due to microgravity causes the displacement of the liquid towards the

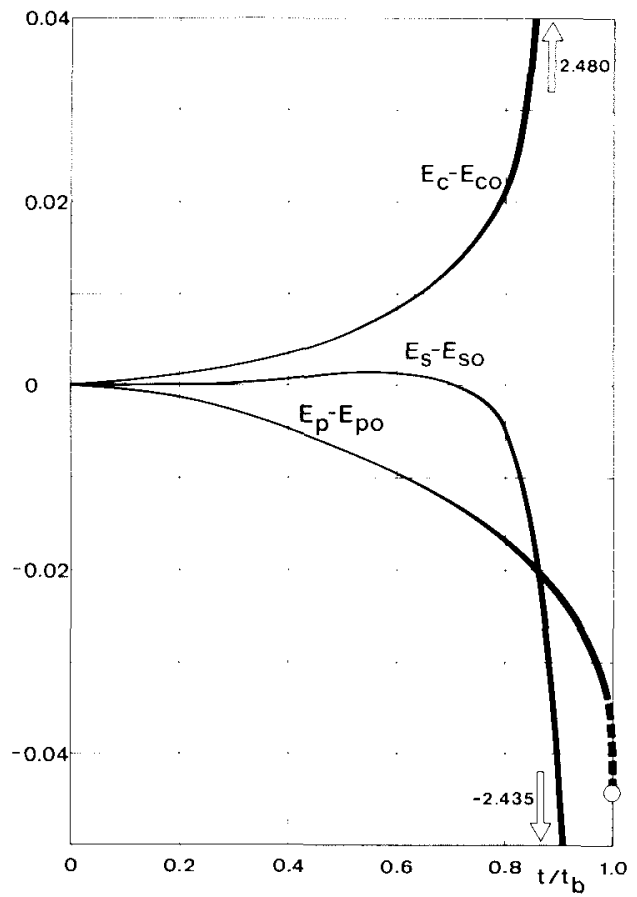

Fig. 7. Variation with time. expressed as a fraction of the breaking time, $t / t_{\mathrm{h}}$, of kinetic, $E_{\mathrm{c}}-E_{\mathrm{c} 0}$, potential, $E_{\mathrm{r}}-E_{\mathrm{r} 0}$. and free surface energies, $E_{\mathrm{s}}-E_{\mathrm{s} 0}$, in the case of an initially cylindrical liquid bridge with $\mathrm{l}=3.1$ and $\mathrm{BO}=0.002$. 
bottom disc: the liquid bridge fattens in its bottom half part and becomes thinner in the upper one. The potential energy decreases and, consequently, the kinetic energy increases as well as the free surface energy *; capillary pressure gradients arise, tending to brake the interface deformation.

Since the slenderness is greater than the corresponding critical value, the deformation of the interface continues, increasing its surface, until the maximum is reached. After this point, the action of the capillary forces tends to strangle the neck of the liquid bridge. Capillary action becomes more effective than the gravitational one and the evolution accelerates: the kinetic energy increases very fast while both potential and free surface energy decrease.

Although the calculations must be stopped before the neck radius vanishes (see fig. 8), it is possible to follow the subsequent evolution. In effect, the volume enclosed between one of the discs and the neck of the liquid bridge varies with time in such a way that close to the breaking time its slope is zero; thence, leaving apart satellite droplets, the volume of the two drops resulting after breakage is known. If the Bond number is small enough these two drops are nearly spherical caps (according to Chesters [14], who studied the profiles of sessile and pendant drops, the perturbation in the radius is, with respect to the sphere, less than $0.01 \%$ when $\mathrm{Bo}=10^{-4}$ and $1 \%$ when $\mathrm{Bo}=$ $10^{-2}$ ) and the final values of both potential and free surface energy, $E_{\mathrm{p} f}$ and $E_{\mathrm{sf}}$ respectively, can be easily calculated through eqs. (27) and (28). In our case the volume of the main drop is 0.8508 times that of the initial cylinder (the main drop is a 1.600 radius spherical cap having its center 1.249

\footnotetext{
* It is well known from hydrostatic studies that the cylindrical shape encompasses a minimum surface area. Thence, any shape close to the cylindrical one, and enclosing the same volume of liquid, presents a larger surface. Amongst these shapes there is one of maximum surface area which is near to that of unstable equilibrium (formally the unstable equilibrium shape is reached when the amount $E_{\mathrm{p}}+E$, is maximum). Then, leaving apart kinetic energy effects, it is clear that breakage will occur when the available potential energy be enough to force the deformation of the interface to go beyond the maximum surface shape: in the opposite case, oscillation will take place.
}

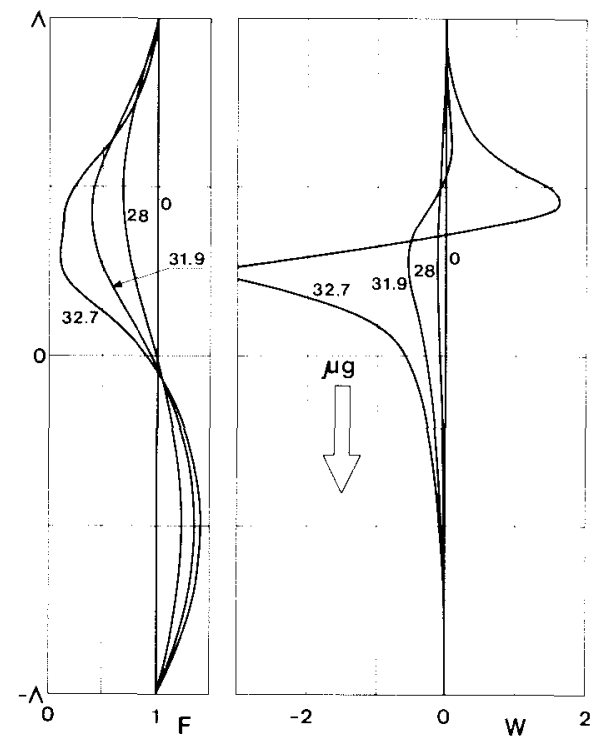

Fig. 8. Variation with time of the shape of the interface. $F$. and the axial velocity field, $W$, of an initially cylindrical liquid bridge with $\lambda=3.1$ and $B o=0.002$. Numbers on the curves indicate elapsed time from the initial time.

over the bottom disc) whereas the volume of the upper drop is 0.1492 times the cylinder volume (a 1.021 radius spherical cap whose center is 0.208 under the top disc). Thence, the following results are obtained:

$E_{\mathrm{pf}}-E_{\mathrm{p} 0}=0.0446, \quad E_{\mathrm{si}}-E_{\mathrm{s} 0}=2.4350$,

therefore, after the last interface shape plotted in fig. 8 , the liquid bridge breaks and the potential energy suddenly falls to its final value (this evolution has been plotted (dashed line) in fig. 7) and oscillates around this final value, the behavior of the kinetic and the free surface energy being similar.

\section{Additional comments on initial conditions}

The main problem in the settlement of numerical initial conditions is the suitability of these conditions from the experimental point of view. The difficulty is placed in the nature of the experimentation with liquid bridges: in the beginning the processes are so slow that it is not an easy task to elucidate the exact initial conditions; certainly, 
some rough initial conditions can be controlled by the experimentalist (the slenderness, the volume of liquid, etc.) but some other characteristics are much more difficult to control (the shape of the interface, the velocity field, etc.).

To study the influence of the initial shape of the interface on the liquid bridge behavior an additional set of calculations have been performed, and the evolution of non-cylindrical axisymmetric liquid bridges with $\Lambda=3.1, \mathrm{Bo}=0.002$ has been calculated. The chosen initial shapes were some of the ones reached by the liquid bridge in its evolution from the cylindrical case, being the initial shapes individualized by the parameter $d_{n}=1-$ $F_{\mathrm{m}}$, which gives the difference between the radius of the discs and the radius of the neck of the liquid bridge. Thence, the numerical process is as follows: the liquid bridge starts from a cylindrical shape and when the deformation of the interface, that is, $d_{n}$, reaches some prefixed value the velocity field is cancelled, taking this point as the initial point for a following run.

The results obtained are collected in table 3 , whereas fig. 9 shows the variation with time of the neck radius for the numerically solved cases. In fig. 10 the variation with the parameter of deformation, $d_{n}$, of both the breaking time and the breaking volume are plotted. Fig. 10 shows that the breaking time decreases with $d_{n}$, the rate of decreasing being small when $d_{n}$ is small, in agreement with the argumentation presented in the preceding section. As the initial deformation grows the breaking time decreases quickly (the liquid

\section{Table 3}

Influence of the initial deformation. $d_{n}$, on the breaking time. $t_{\mathrm{h}}$. and the hreaking volume. $V_{\mathrm{p}}$. of liquid bridges. having cylindrical volume, with $A=3.1$, Bo $=0.002$

\begin{tabular}{lll}
\hline$d_{\mathrm{n}}$ & $t_{\mathrm{h}}$ & $V_{\mathrm{p}}$ \\
\hline 0 & 32.7 & 0.8508 \\
0.02 & 32.2 & 0.8508 \\
0.04 & 31.4 & 0.8508 \\
0.10 & 25.6 & 0.8509 \\
0.20 & 14.0 & 0.8525 \\
0.30 & 8.0 & 0.8542 \\
0.40 & 4.9 & 0.8587 \\
0.50 & 2.9 & 0.8529 \\
\hline
\end{tabular}

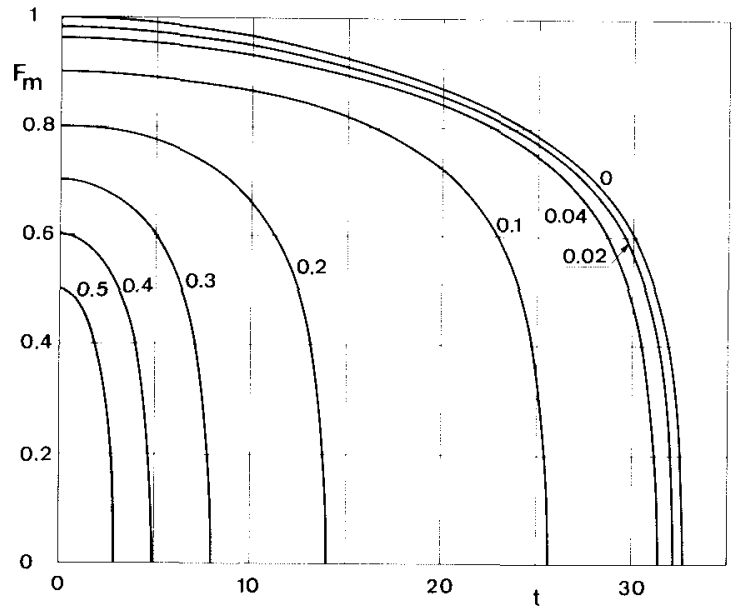

Fig. 9. Variation with time, $t$, of neck radius of the liquid bridge, $F_{\mathrm{m}}$, in the case of a liquid bridge with $\Lambda=3.1$ and $\mathrm{Bo}=0.002$. Numbers on the curves indicate values of the initial deformation, $d_{n}$.

bridge would break even in the case $\mathrm{Bo}=0$ ) and for large values of $d_{n}$ the rate of decreasing becomes small again because inertia effects are more and more important as $d_{1}$ increases. Concerning the breaking volume the results obtained are the

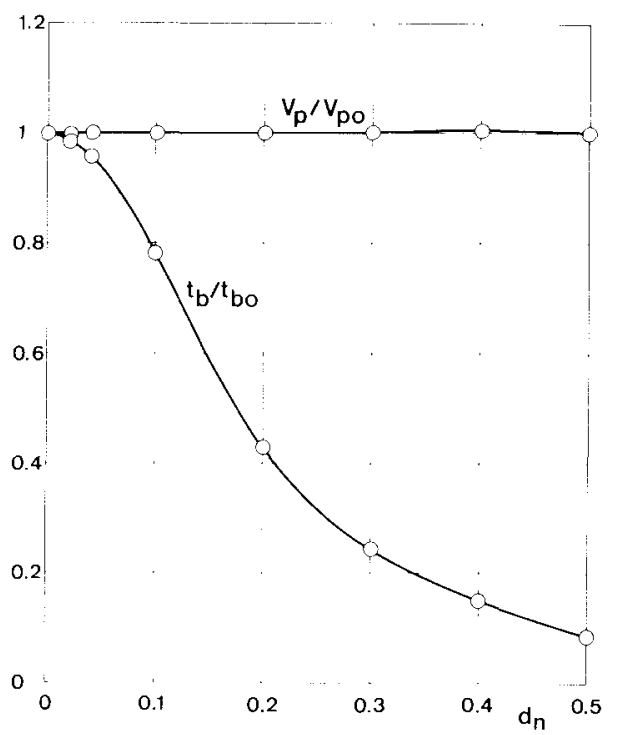

Fig. 10. Breaking volume and breaking time, expressed as fractions of the corresponding values for the initially cylindrical liquid bridge. $V_{\mathrm{p}} / V_{\mathrm{p} u}$ and $t_{\mathrm{b}} / t_{\mathrm{b}(0)}$. respectively, versus initial deformation, $d_{n}$. Results correspond to a liquid bridge with $A=3.1$ and $B \circ=0.002$. 
more surprising: the initial deformation does not change the value of $V_{p}$, being the difference between the two calculated extreme values of $V_{p}$ less than $1 \%$ of the breaking volume obtained when $d_{n}=0$ (cylindrical shape). This result agrees with previous results published elsewhere for the case $B_{0}=0[8]$ in the sense that the breaking volume of liquid bridges having cylindrical volume depends on the slenderness of the liquid bridge, but not on the magnitude of the initial deformation of the interface.

Finally, it must be pointed out that the results presented in this section correspond to liquid bridges having slendernesses greater than the critical value, and, consequently, the evolution will be always of breakage, independently of the initial conditions. If $\Lambda<\Lambda_{c r}$ the results are rather different, and even the character of the evolution (oscillation or breakage) could change depending on the value of the initial deformation of the interface.

\section{Acknowledgement}

This work has been supported by the Spanish National Committee for Space Research (CONIE) under a contract with the Polytechnic University of Madrid (UPM).

\section{References}

[1] J.R. Carruthers and M. Grasso, J. Appl. Phys. 43 (1972) 436.

[2] S.R. Coriell. S.C. Hardy and M.R. Cordes, J. Colloid Interface Sci. 60 (1977) 126

[3] S.R. Coriell and M.R. Cordes, J. Crystal Growth 42 (1977) 466.

[4] E.A. Boucher and M.J.B. Evans, J. Colloid Interface Sci. 75 (1980) 409.

[5] L.H. Ungar and R.A. Brown, Phil. Trans. Roy Soc. London A306 (1982) 347

[6] J.M. Vega and J.M. Perales, in: Material Sciences in Space, Proc. 4th European Symp.. ESA Special SP-191 (European Space Agency, Paris, 1983).

[7] H.C. Lee, IBM J. Res. Develop. 18 (1974) 364

[8] J. Meseguer, J. Fluid Mech. 130 (1983) 123.

[9] P.J. Roache, Computational Fluid Dynamics (Hermosa. Albuquerque, NM, 1972).

[10] A.R. Mitchell and D.F. Griffiths, The Finite Difference Method in Partial Differential Equations (Wiley, New York, 1980).

[11] W. Knabe and D. Eilers, Acta Astronautica 9 (1982) 187

[12] J.R. Carruthers and M. Grasso, J. Crystal Growth 13/14 (1972) 611 .

[13] A. Sanz and I. Martinez, J. Colloid Interface Sci. 93 (1983) 235.

[14] A.K. Chesters, J. Fluid Mech. 81 (1977) 609. 